\title{
DILATACIÓN HIDRÁULICA (URETEROMAT): URETEROSCOPIA EFICAZ Y SEGURA
}

Luciano Cima', José Ignacio Iglesias Prieto, Rodolfo Orozco Fariñas, Jorge Massarra Halabi, José Mª Mancebo Gómez y Enrique Pérez-Castro Ellendt

Unidad de Urologia. Clinica La Luz. Madrid. España.

'Becario de Sanatorio Parque Salta. Argentina.

Resumen.- OBJETIVO: Tras la descripción y estandarización de la técnica de ureteroscopia en 1979, se han desarrollado varios métodos para conseguir un acceso ureteral no traumático, simple, eficiente y rápido. La dilatación hidráulica del uréter fue posible en 1986, con el desarrollo de Ureteromat. Se evaluó la efectividad de la dilatación ureteral hidráulica (Ureteromat) para el acceso ureteral y el procedimiento endoscopico posterior.

MÉTODO: Se evaluaron las 100 últimas ureteroscopias llevadas a cabo desde febrero hasta octubre del 2006. En todas las ureteroscopias se utilizo un ureteroscopio semirígido de $9.8 \mathrm{Fr}$ y en todos los procedimientos se realizó dilatación hidráulica (Ureteromat) como se viene realizando desde 1986.
RESULTADOS: Del total de los casos, 92 se indicaron por patología litiasica, 7 fueron exploradoras y 1 por estrechez ureteral. En 94 pacientes el procedimiento se llevó a cabo con dilatación hidráulica exclusivamente, en 2 casos se realizó además dilatación con catéter balón, en 3 pacientes dilatación del meato con pinza y sólo en 1 procedimiento no fué posible por un stop ureteral.

CONCLUSIÓN: La bomba de irrigación Ureteromat garantiza una excelente dilatación hidráulica del meato ureteral y del trayecto intramural del uréter. La visibilidad es muy buena en todo momento, haciendo de la ureteroscopia un método simple, eficaz, no traumático y rápido.

Palabras clave: Ureteroscopia. Dilatación hidráulica. Ureteromat.

Summary.- OBJECTIVES: After the description and standardization of the technique of ureteroscopy in 1979, several methods to obtain a nontraumatic, simple, efficient and fast ureteral acce-ss have been developed. The hydraulic expansion of the ureter was possible in 1986, with the development of ureteromat. We evalua-te the effectiveness of hydraulic ureteral expansion (Ureteromat) for ureteral access and the later endos-copic procedures.

METHOD: The last 100 ureteroscopy carried out from February to October 2006 were evaluated. We used a semi-rigid 9.8 Fr. ureteroscope and hydraulic expansion (Ureteromat) was performed in the same way in all procedures since 1986.

RESULTS: Of the total of cases, 92 were indicated for lithiasic pathology, 7 were exploratory and 7 for ureteral 
stricture. In 94 patients the procedure was carried out with hydraulic expansion alone, in 2 cases we used a balloon catheter, in 3 patients expansion of the mea-tus was done with forceps and 1 procedure was not possible due to an ureteral stop.

CONCLUSION: The Ureteromat pump guarantees an excellent hydraulic expansion of the ureteral meatus and the passage of the intramural ureter. The visibility is very good at any moment of the procedure, making ureteroscopy a simple, effective, non traumatic and fast method.

Keywords: Ureteroscopy. Hidraulic expansion.

Ureteromat.

\section{INTRODUCCIÓN}

La primera publicación de la dilatación ureteral, la realizó Pawick en 1901, al tratar las estrecheces ureterales secundarias a tuberculosis, con bujías introducidas por cistoscopia (1). Tras la descripción y estandarización de la técnica de la ureteroscopia en 1979 (2), una de las principales dificultades, ha sido conseguir un acceso ureteral no traumático, simple, eficiente y rápido. Desde entonces se han desarrollados varios métodos (bujías, catéter balón, catéter teflonado, etc.), según la experiencia de diferentes grupos, para facilitar este proceder (3-7).

La dilatación ureteral es necesaria para la introducción del ureteroscopio en el meato ureteral y el segmento intramural. Con el advenimiento de ureteroscopios de menor calibre (6-7 Ch), esta puede obviarse en la ureteroscopia diagnóstica (4), pero es necesaria en la mayoría de las ureterosco-

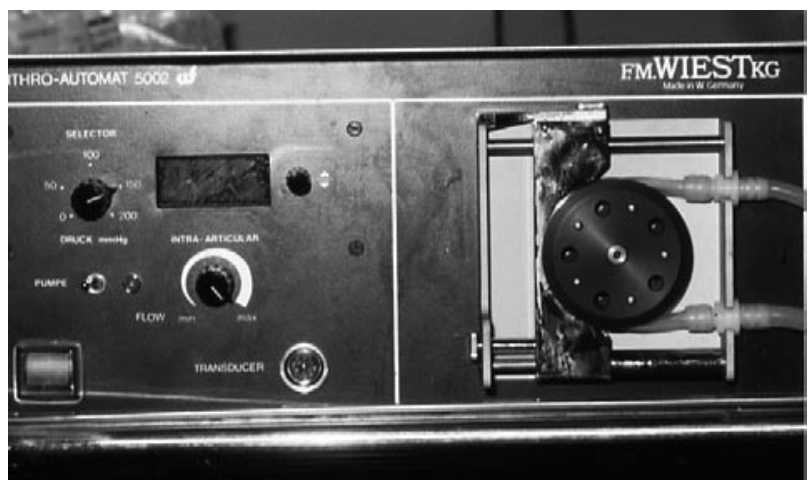

FIGURA 1. Ureteromat (Wiest Germany) pias terapéuticas (litotricia endoscópica o resección transureteral) (3-4-8), relacionando algunos autores el porcentaje de éxito del tratamiento a la dilatación ureteral previa (9).

La dilatación hidráulica del uréter fue posible en 1986, con el desarrollo de Ureteromat (Wiest Germany) (Figura 1) $(10,11)$, basándose en el sistema de irrigación que utilizan los artroscopistas y la necesidad de aumentar en determinados momentos el flujo de irrigación durante la ureteroscopia con inyecciones salinas por el canal lateral o la compresión manual de la bolsa de irrigación por un ayudante (Figura 1).

\section{OBJETIVO}

Evaluar la efectividad de la dilatación ureteral hidráulica (Ureteromat) para el acceso ureteral y el procedimiento endoscópico posterior.

\section{MATERIAL Y MÉTODOS}

Se realizó un estudio retrospectivo, descriptivo por medio de la revisión de historias clínicas, de las 100 últimas ureteroscopias llevadas a cabo en la Clínica La Luz, desde febrero hasta octubre del 2006.

De manera protocolar, los pacientes fueron evaluados con anamnesis y exploración física, analítica general con estudio de coagulacion, urocultivo y evaluación cardiológica preanestésica. Todos los pacientes tienen urografía previa y no se realiza preparación intestinal a ningún paciente.

Todas las ureteroscopias se realizaron con ureteroscopio semirígido de 9.8 Fr. La posición del paciente fue en todos los casos la descrita originalmente para la técnica (2), posición de litotomía modificada, con la pierna contralateral al uréter a explorar baja y en leve abducción. Se realizó anestesia regional con sedacion en todos los casos otorgándose el alta sanatorial a las 24 horas.

En todos los procedimientos se realizó dilatación hidráulica (Ureteromat) como se viene realizando desde 1986. El Ureteromat (Figuras 2 y 3 ) es una bomba impulsora de líquido creada para aportar flujo y presión controlado, en cualquier instrumento endoscópico. Permite flujos máximos de $400 \mathrm{cc} /$ minuto y presión máxima de $200 \mathrm{mmHg}$, con dos válvulas de seguridad, que desconectan el mecanismo de inyección al alcanzar la presión y flujo preseleccionado. 

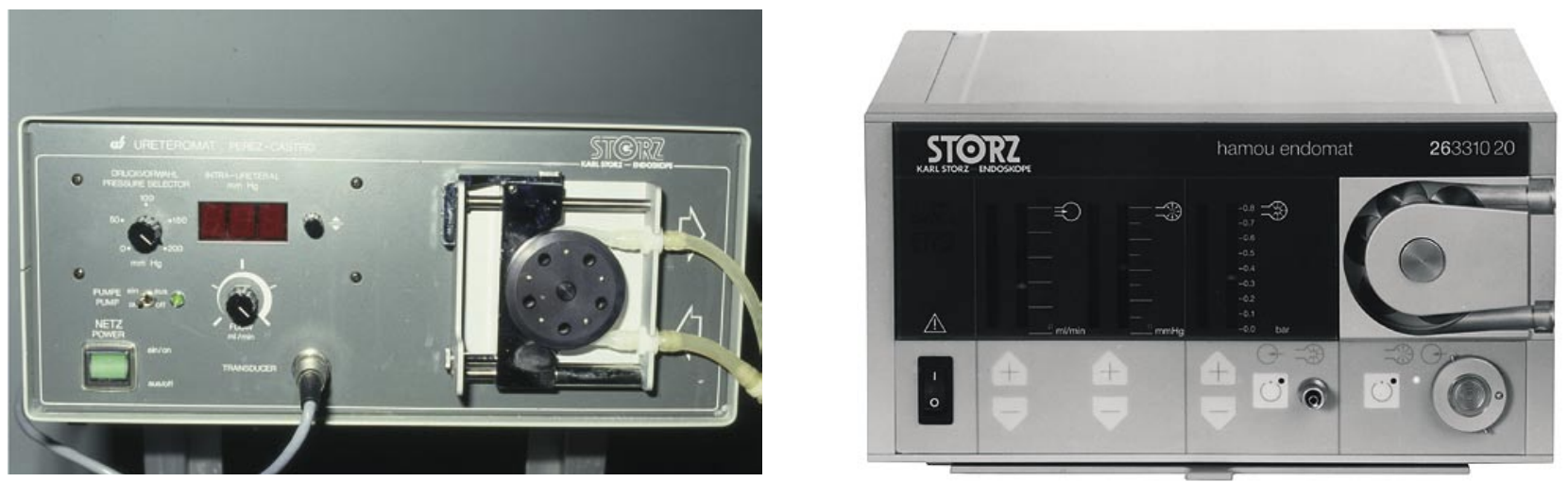

FIGURAS 2 y 3 . Ureteromat.

Se evaluó la efectividad de este procedimiento mediante los casos resueltos sin necesidad de otra técnica de dilatación ureteral y corroborando al mismo tiempo la ausencia de complicaciones propias del acceso ureteral.

\section{RESULTADOS}

Desde febrero hasta octubre de 2006 se realizaron 100 ureteroscopias en 57 varones y 43 mujeres. La edad promedio de la población fue de 52 años (rango $16-80$ ).

Del total de los procedimientos, 92 se indicaron por patología litiásica, 7 fueron exploradoras (de estas 2 por hematuria), 1 por estrechez ureteral. En 94 pacientes se realizó dilatación hidráulica unicamente, pudiendo conseguir un buen acceso ureteral y la resolución del caso. En los que no se consiguió una buena dilatación con Ureteromat, se realizó ademas dilatación con catéter balón en 2 casos, dilatación con pinza en 3 casos ( 2 pacientes con estrechez del meato y 1 con RTUV previa) y sólo 1 procedimiento no fue posible por un stop ureteral (Tabla I).

Entre los pacientes con patología litíasica (Tabla II), el tamaño promedio de esta fué de 8.94 $\mathrm{mm}$ (rango $4-20 \mathrm{~mm}$ ), de las cuales 58 se pudieron extraer con pinza (tamaño promedio de $7.11 \mathrm{~mm}$ ) y 34 requirieron litotricia con láser-holmio (tamaño promedio de $8.76 \mathrm{~mm}$ ).

\section{TABLA I. 100 URETEROSCOPIAS (URS). POBLACIÓN EN ESTUDIO, CARACTERÍSTICAS GENERALES.}

\begin{tabular}{|l|l|}
\hline \multirow{2}{*}{ Edad } & 52 años (rango 16-80) \\
\hline \multirow{2}{*}{ Sexo } & $57 \mathrm{~V}$ \\
\hline \multirow{2}{*}{ Indicación } & 92 por litiasis \\
\cline { 2 - 2 } & 7 Exploradora \\
\cline { 2 - 2 } & 1 por estrechez ureteral \\
\hline Dilatación Ureteral & 94 dilatación hidráulica (Ureteromat) \\
\cline { 2 - 2 } & 3 dilatación con pinza \\
\cline { 2 - 2 } & 2 dilatación con catéter balón \\
\cline { 2 - 2 } & 1 no posible (stop) \\
\hline Resultados & 99 URS eficaces \\
\hline Complicación & Ninguna \\
\hline
\end{tabular}


La ubicación litiásica más frecuente fue del lado izquierdo (57 casos), siendo en 30 pacientes en el uréter pelviano ( 2 calles litiásicas), en 17 yuxtavesical, en 7 en uréter sacro, 2 en uréter lumbar y 1 litiasis piélica en un riñón ectópico. En el lado derecho (35 casos), 20 pacientes presentaron litiasis en uréter pelviano ( 3 calles litiásicas), 9 en uréter yuxtavesical y 6 en uréter sacro.

De las 100 ureteroscopias, 10 pacientes tenían colocado un catéter doble J previo al procedimiento, 5 habían realizado ESWL (Extracorporeal Shockwave Lithotripsy) previa y 1 NLP (Nefrolitotomía Percutánea) con colocación de catéter doble J.

En sólo 14 pacientes se colocó catéter doble J post ureteroscopia (8 litotricias con láser-holmium, 4 extracciónes litiásicas con pinza y 2 sólo ureteroscopia exploradora) en 51 pacientes se colocó un catéter ureteral post ureteroscopia, que se retiró al alta sanatorial del paciente ( $24 \mathrm{hs}$ ) y en 35 casos no se dejó ningun tipo de cateter.

No se evidenció ninguna complicación, tanto intraoperatoria como en el post operatorio, consiguiéndose en 94 pacientes un buen acceso ureteral mediante Ureteromat y en los mismos la resolución del caso.

\section{DISCUSIÓN}

Actualmente existen distintos sistemas de dilatación del trayecto intramural del uréter, distinguiéndose los métodos mecánicos (catéter, telescopados, vainas teflonadas, bujías, sistema olivar y catéter balón) e hidráulico (Ureteromat).

Con excepción del catéter balón, el resto de los sistemas requieren del paso previo del material de

TABLA II. 92 URS POR LITIASIS. CARACTERISTICAS DE URS POR LITIASIS. (* EN LAS 100 URSI.

\begin{tabular}{|c|c|c|}
\hline Litiásis: tamaño promedio total (mm) & \multicolumn{2}{|c|}{8.94 (rango $4-20 \mathrm{~mm}$ ) } \\
\hline \multirow{10}{*}{ Ubicación } & \multirow{6}{*}{57 Izquierda } & 30 Ureter pelviano \\
\hline & & (2 calles) \\
\hline & & 17 Yuxtavesical \\
\hline & & 7 Uréter sacro \\
\hline & & 2 Uréter lumbar \\
\hline & & 1 Riñón ectópico \\
\hline & \multirow{4}{*}{35 Derecha } & 20 Uréter pelviano \\
\hline & & (3 calles) \\
\hline & & 9 Yuxtavesical \\
\hline & & 6 Uréter sacro \\
\hline \multirow{2}{*}{$\begin{array}{c}\text { Tratamiento y } \\
\text { tamaño promedio }(\mathrm{mm})\end{array}$} & \multirow[t]{2}{*}{58 extracción con pinza $(7.11 \mathrm{~mm})$} & 34 Láser-Holmium \\
\hline & & $(8.76 \mathrm{~mm})$ \\
\hline \multirow{5}{*}{$\begin{array}{l}\text { Post - URS } \\
{ }^{*}(n: 100)\end{array}$} & \multirow{3}{*}{14 Colocación de JJ } & 8 Laser - Holmium \\
\hline & & 4 extracción con pinzo \\
\hline & & 1 Exploradora \\
\hline & \multicolumn{2}{|c|}{51 catéter ureteral (24 hs) } \\
\hline & \multicolumn{2}{|c|}{35 nada } \\
\hline
\end{tabular}


dilatación por cistoscopia, siendo en algunos casos necesario retirar la óptica para el paso de los mismos, realizando el procedimiento a ciegas sobre un alambre guía y bajo control radiológico. Este proceder, puede provocar lesiones en uretra, cuello vesical y ostium ureteral $(4,5)$.

En el caso de usar catéter balón, se debe realizar presión con jeringas-manométricas, con aumento progresivo de la presión para preservar la integridad de las fibras musculares, evitando la posterior fibrosis y retracción (4,5-12). Es importante destacar la dificultad que se presenta con este procedimiento, ante litiasis yuxtavesical o calles litiasicas y la particularidad que muchos de estos cateteres estan diseñados para un solo uso. En nuestro grupo de estudio, se presentaron 26 pacientes con litiasis yuxtavesical y 5 con calle litiásica. En ningún caso se vió imposibilitado el acceso ureteral y tampoco se evidenció la migración de fragmentos al utilizar el flujo máximo de la bomba.

Con la dilatación hidráulica, la ureteroscopia se convierte en un procedimiento de un solo gesto $(13,14)$. Al obviar la cistoscopia previa para el cateterismo ureteral y el trabajo dedicado a la dilatación de la porción intramural del uréter, el tiempo de acceso al uréter se reduce notablemente.

El flujo conseguido mediante la bomba de irrigación, permite un buen avance intrauretral del ureteroscopio, manteniendo una visión intraureteral superior a la alcanzada con la irrigación simple, sin bomba. Una vez en vejiga, tras localizar el meato ureteral, este puede cateterizarse con un catéter de 3 o $5 \mathrm{fr}$. Con el catéter posicionado o no, en el segmento distal del uréter, el propio flujo hidráulico produce la apertura del ostium, permitiendo un acceso con buena y constante visión de la luz ureteral que reduce los riesgos propios de esta maniobra. De esta manera el avance del ureteroscopio no presenta dificultad, haciendo de la ureteroscopia un procedimiento seguro, sobretodo ante la presencia de bucles ureterales, los cuales en su gran mayoría corrige.

La ureteroscopia es un procedimiento invasivo y por lo tanto no exento de complicaciones. Dentro de estas pueden distinguirse entre: mayores (avulsión o perforación ureteral, sepsis) y menores (hematuria, falsa vía, fiebre) (4). En la serie del estudio, se evidenció una tasa de éxito del $94 \%$ (94 URS) para la dilatación hidráulica con resolución del caso, no observándose ninguna complicación, tanto en el acto operatorio como en el post-operatorio. Al igual que nosotros, Eshghi y cols. (14), con una serie de 48 pacientes demuestra que la dilatación hidráulica es el método menos traumático para el acceso ureteral, garantizando una tasa de éxito del $97 \%$ (47 pacientes) para el tratamiento con ureteroscopia.

Existe una idea de que se trabaja con altas presiones ureterales que podrian comprometer el riñon, pero diversos estudios han demostrado que dicha presión no es peligrosa si no se prolonga en el tiempo (15). De todas maneras, si la ureteroscopia va a extenderse demasiado, la presión intraureteral puede evitarse con la introducción de un catéter ureteral y una sonda vesical $(8-10 \mathrm{Fr}$ ) en paralelo al ureteroscopio.

\section{CONCLUSIÓN}

La bomba de irrigación Ureteromat garantiza una excelente dilatación hidráulica del meato ureteral y el trayecto intramural del uréter. La visibilidad es muy buena en todo momento del procedimiento, haciendo de la ureteroscopia un método simple, no traumático, eficiente y rápido. La ausencia de complicaciones demuestra que no existen riesgos de utilizar presión hidraulica intraureteral.

\section{BIBLIOGRAFIA y LECTURAS RECOMENDADAS ( ${ }^{*}$ lectura de interés $y^{* *}$ lectura fundamental)}

1. MORRIS, H.: "Surgical disease of kidney and uréter". London, Gassell, 1901.

2. PEREZ-CASTRO, E.; MARTINEZ-PIÑEIRO, J.A.: "La ureteroscopia transuretral. Un actual proceder urológico". Arch. Esp. Urol., 5: 1, 1980.

**3. PEREZ-CASTRO, E.; IGLESIAS PRIETO, J.I.: "Ureteral dilatation: indications and techniques". Smith's text- book of endourology, chapter 29. St. Louis: Quality Medical Publishing, 1996.

4. VICENTE, J.: "Tratado de endourología". Cap. 3: Cirugía endoscopica del uréter. Ed. Pulso 1996.

**5. IGLESIAS PRIETO, J.I.; MANCEBO GOMEZ, J.M.; MASSARRA HALABI, J. y cols.: "Técnicas de acceso endoscopico ureteral". Arch. Esp. Urol., 48: 813, 1995.

6. ZULUGA GOMEZ, A.; ARRABAL MARTIN, M.; GUTIERREZ TEJERO, F. y cols.: "Ureterorrenoscopia diagnóstica y terapéutica". Clínicas urológicas de la complutense: endoscopia, laparoscopia y terapia minimamente invasiva en urología, Vol. 11, 2005.

7. CAPITAN MANSON, C.; MARTINEZ SILVA, V.; PAMPLONA CASAMAYOR, M. y cols.: "Tratamiento endourológicos de la litiásis ureteral". Clínicas urológicas de la complutense: endoscopia, laparoscopia y terapia minimamente invasiva en urología, Vol. 11, 2005. 
8. ESCOBAR, P.; REY, M.; LÓPEZ, J.R. y cols.: "Tratamiento endoscópico de los cálculos ureterales. Endourología y ondas de choque”. Raúl Clemente Editor, Valencia, Venezuela, 143-205, 1987.

*9. RODRIGUES NETTO, N. Jr.; CASERTA LEMOS, G.; LEVI D'ANCONA, C.A. y cols.: "Is routine dilation of the ureter necessary for ureteroscopy?". Eur. Urol., 17: 269, 1990.

***10. PEREZ-CASTRO, E.; MANCEBO, J.M.; MASARRA, J.: "Dilatación hidráulica con bomba impulsora de líquido irrigante. Nuevo aparataje en ureterorenoscopia". Arch. Esp. Urol., 39: 3, 1986.

**11. PEREZ-CASTRO, E.: "Ureteromat. Method to facilitate ureterorenoscopy and avoid dilatation". Urol. Clin. North Am., 15: 315, 1988.

*12. *THOMAS, R.: "Balloon dilatation of the ureteral orifice for ureteroscopy". Smith: Controversies in Endourology. Philadelphia, WB Saunders, Pp: 315-320, 1995.

*13. PEREZ-CASTRO, E.; IGLESIAS PRIETO, J.I.: "No dilatation of the ureteral orifice and the use of hydraulic pump: ureteromat. Controversies in endourology". Philadelphia: WB Saunders, 324, 1995.

**14. ESHGHI, M.: "Pressure-controlled hydraulic dilation of the ureter: One-step ureteroscopy". J. Urol., 140: 950, 1988. 